\title{
ACF7 regulates colonic permeability
}

\author{
YONG LIANG, CHENZHANG SHI, JUN YANG, HONGQI CHEN, YANG XIA, \\ PENG ZHANG, FENG WANG, HUAZHONG HAN and HUANLONG QIN
}

\author{
Department of Surgery, The Sixth People's Hospital Affiliated to \\ Shanghai Jiaotong University, Shanghai 200233, P.R. China
}

Received November 27, 2012; Accepted February 4, 2013

DOI: $10.3892 / \mathrm{ijmm} .2013 .1284$

\begin{abstract}
Colonic paracellular permeability is regulated by various factors, including dynamics of the cytoskeleton. Recently, ACF7 has been found to play a critical role in cytoskeletal dynamics as an essential integrator. To elucidate the physiological importance of ACF7 and paracellular permeability, we conditionally knocked out ACF7 in the intestinal mucosa of mice. Histopathological findings indicated that ACF7 deficiency resulted in significant interstitial proliferation and columnar epithelial cell rearrangement. Decreased colonic paracellular permeability was detected using a Ussing chamber and the FITC-inulin method. In order to clarify the underlying mechanism, we further analyzed the expression levels of three important tight junction proteins. Downregulation of ZO-1, occludin and claudin-1 was identified. Immunofluorescence provided strong evidence that ZO-1, occludin and claudin-1 were weakly stained. We hypothesized that ACF7 regulates cytoskeleton dynamics to alter mucosal epithelial arrangement and colonic paracellular permeability.
\end{abstract}

\section{Introduction}

The physiological function of the intestinal barrier regulates selective passage from the gut lumen, i.e. the transport of ions or small molecules but not large molecules and microorganisms (1). The intestinal barrier is composed of both a cellular barrier and a paracellular barrier. The paracellular barrier largely consists of the tight junction (TJ) between epithelial cells (1). The TJ is composed of tight junction proteins (TJPs) which is a group of proteins with various functions and molecular structures. The TJPs regulate the function of the intestinal barrier through their expression level and functional status (2). A change in the levels of TJPs is considered as a marker of impaired intestinal barrier and increased mucosal permeability in many diseases (3).

Correspondence to: Professor Huanlong Qin, Department of Surgery, The Sixth People's Hospital Affiliated to Shanghai Jiaotong University, 600 Yishan Road, Shanghai 200233, P.R. China

E-mail: hlqin10@163.com

Key words: ACF7 conditional knockout, Ussing chamber, FITCinulin, colonic paracellular permeability, tight junction proteins
Previous studies have shown that TJPs are involved in the regulation of intestinal permeability (4). Recently, changes in the cytoskeleton dynamics have also been noted in the alteration of intestinal permeability (5). It has been shown that in the T84 colon cancer cell line, disrupted F-actin results in increased paracellular permeability (6). The regulation of intestinal permeability is a dynamic system in which the mobility of intestinal epithelial cells is critically involved. It has been noted that their interaction is the key to the regulation of intestinal permeability (4). However, little is known concerning the regulation of intestinal paracellular permeability in regard to the interaction among TJPs and cytoskeleton proteins.

Microtubes (MTs) are required for cellular mobility and maintenance of cellular morphology (7). Dynamic cytoskeleton formation by microfilaments and MTs determines cellular mobility and cell shape (8). Kodama et al (9) demonstrated that ACF7 is critically required in MT-microfilament dynamics. ACF7 can stabilize downstream cytoskeleton structure by either direct binding to MTs or forming links between MTs and microfilaments.

In this study, we employed a conditional gene targeting method to ablate the ACF7 gene in colonic mucosa in order to create ACF7-conditional knockout (cKO) mice. We also employed FITC-inulin, an effective tracer for paracellular permeability assay (10). By utilizing a Ussing chamber and FITC-inulin, we confirmed this mouse as a murine model for decreased colonic paracellular permeability. Hematoxylin and eosin (H\&E) staining revealed histopatholigical changes in the mucosal epithelial arrangement and interstitial proliferation. Since TJPs are vital cytoskeletal proteins, we determined the transcription and expression levels of ZO-1, occludin and claudin-1, which are typical members of the TJPs. Our findings revealed that the three TJPs were downregulated in this ACF7-cKO model. Immunofluorescence for the three proteins also found weak staining for ACF7 in the $\mathrm{cKO}$ mice.

Therefore, based on this significant evidence we hypothesize that ACF7 regulates tight junction protein expression, changes mucosal epithelial arrangement and consequently alters colonic paracellular permeability.

\section{Materials and methods}

Generation of ACF7-cKO mice. Due to the lethal effect of ACF7 deficiency in conventional knockout models (11), we 
Table I. Primers for qRT-PCR of GAPDH, occludin, claudin-1 and ZO-1.

\begin{tabular}{lllc}
\hline Gene & Primers & \multicolumn{1}{c}{ Sequence (5'-3') } & Products (bp) \\
\hline GAPDH & Ga-F & AGGTTGTCTCCTGCGACTTCA & 143 \\
(internal control) & Ga-R & GAGGTCCACCACTCTGTTGCT & 168 \\
Occludin & Oc-F & GCAGCCTTCTGCTTCATCG & 163 \\
& Oc-R & CGTCGGGTTCACTCCCATTA & 271 \\
Claudin-1 & Ca-F & TGGGTTTCATCCTGGCTTCT & \\
& Ca-R & TGTATCTGCCCGGTGCTTT & \\
ZO-1 & Zo-F & TCACGATCTCCTGACCAACG & \\
& Zo-R & GGCTGACGGGTAAATCCACA & \\
\hline
\end{tabular}

F, forward; R, reverse.

crossed $A C F 7^{f l / f l}$ mice (12) and Vil-cre transgenic mice to generate $\mathrm{cKO}$ mice. $\mathrm{ACF} 7^{\mathrm{f} / \mathrm{l}}$ mice were kindly donated by Xiaoyang $\mathrm{Wu}$ of the Howard Hughes Medical Institute and Laboratory of Mammalian Cell Biology and Development, Rockefeller University, New York, NY, USA. B6.SJL-Tg(Vilcre) $997 \mathrm{Gum} / \mathrm{J}$ mice of C57BL/6 background were purchased from Jackson Laboratory (Bar Harbor, ME, USA).

Vil-cre transgenic mice were identified by PCR using DNA extracted from the peripheral blood of 4-week-old transgenic mice using PCR primers: forward, 5'-GTGTGG GACAGAGAACAAACC-3' and reverse, 5'-ACATCTTCA GGTTCTGCG GG-3'. The PCR reaction generated a 1,100-bp product. Excision efficiency was assessed by PCR using the following primers: upstream, 5'-AAAGAAACGGAAATA CTGGCC-3' and downstream, 5'-GCAGCTTAATTCTGCC AAATTC-3'; with 650- and 700-bp PCR products, respectively. ACF7-cKO mice were generated by crossing F2 in a specific pathogen-free (SPF) environment. The Vil-cre and ACF7 genes were synthesized by Sangon Biotech Co., Ltd. (Shanghai, China).

All mice were maintained in an SPF facility with a regular light cycle (12 h light and $12 \mathrm{~h}$ dark) at a controlled temperature $\left(23 \pm 1^{\circ} \mathrm{C}\right)$ and relative humidity $(50 \%)$. Food (SCXK 2008-0016; Shanghai Super B\&K Laboratory Animal Corp., Ltd., Shanghai, China) and water were freely available to mice throughout the experimental period. The Sixth People's Hospital Animal Care and Utilization Committee Affiliated to Shanghai Jiaotong University approved all experiments relating to ethical standards.

$H \& E$ staining. Intestinal samples were fixed in $10 \%$ formalin for $12 \mathrm{~h}$, and then embedded in paraffin. Sections $(3-\mu \mathrm{m})$ were stained with H\&E and evaluated by a pathologist. Microscopy of $x 200$ and $x 400$ fields was applied to capture typical images using a DSY5000X microscope and Nikon D200 camera.

Measurement of colonic mucosal paracellular permeability by Ussing chamber and FITC-inulin. Mice were sacrificed at 8-12 weeks of age by cervical dislocation. The proximal segment of the colon was dissected, of which the mucosa was stripped from the muscular layer within $10 \mathrm{~min}$. The mucosa was mounted in Lucite chambers exposing the mucosal and submucosa surfaces to $10 \mathrm{ml}$ of oxygenated Krebs buffer. The buffer was maintained at $37^{\circ} \mathrm{C}$ by a heated water jacket and circulated by $\mathrm{CO}_{2} / \mathrm{O}_{2}(13)$.

Non-absorbable tracer molecule FITC-inulin $(1.0 \mathrm{mg} / \mathrm{ml})$ (MW 5,000 kDa) (Sigma-Aldrich, USA) was added to the mucosal side with a spread mucosa area of $0.3 \mathrm{~cm}^{2}$. Buffer samples of $200 \mu \mathrm{l}$ from the submucosa side were collected at 30, 60, 90 and $120 \mathrm{~min}$, and samples were analyzed for fluorescence in black walled 96-well plates (Greiner, Germany) using a Varioskan Flash Scanner (Thermo Scientific, Finland) with excitation at $485 \mathrm{~nm}$ and emission at $530 \mathrm{~nm}(14,15)$.

RNA extraction and $q R T-P C R$. ACF7-cKO and $\mathrm{ACF} 7^{\mathrm{A} / \mathrm{f}}$ mice were sacrificed at 12 weeks of age by cervical dislocation. A segment of the colon was dissected at $1.5 \mathrm{~cm}$ distal to the cecum. Each colon sample was scissored to expose its interior on clean slides. The mucosa was scraped from the muscular layer and preserved in RNAlater (Invitrogen, USA) solution at $-20^{\circ} \mathrm{C}$ for further analysis.

We used qRT-PCR to estimate the expression of TJPs (ZO-1, occludin and claudin-1). Briefly, $30 \mathrm{mg}$ of the mucosa scraps was homogenized for RNA extraction. RNA was extracted using a total RNA extraction kit (SLNCO, China), followed by reverse transcription using a qPCR-RT kit (Toyobo Co., Ltd., Japan). cDNA was then evaluated by qRT-PCR using Real-Time PCR Master Mix (Toyobo Co., Ltd.) in a FTC-3000 PCR Cycler (Funglyn Biotech, Inc., Canada) using the primers (Generay Biotech Co, Ltd., China) listed in Table I. Denaturation, annealing and extension temperatures were set at $95^{\circ} \mathrm{C}, 60^{\circ} \mathrm{C}$ and $68^{\circ} \mathrm{C}$ for $30 \mathrm{sec}$ each, respectively, for 40 cycles according to routine procedures.

Western blot analysis. Cytoplasmic proteins were extracted from mucosal samples from ACF7-cKO and $\mathrm{ACF} 7^{\mathrm{fl} / \mathrm{fl}}$ mice using a nucleic/plasma protein extraction kit (ViaGene, USA). After quantitation, cytoplasmic protein was then separated by SDS-polyacrylamide gel electrophoresis (SDS-PAGE). Samples were separated on 5\% SDS-PAGE for ACF7 protein and on 10\% SDS-PAGE for ZO-1, occludin and claudin-1 for $1 \mathrm{~h}$ respectively (Bio-Rad, USA) and transferred onto nitrocellulose membranes (Millipore, USA) at $4^{\circ} \mathrm{C}, 200 \mathrm{~mA}$, overnight for ACF7 and $1 \mathrm{~h}$ for ZO-1, occludin and claudin-1. 
A

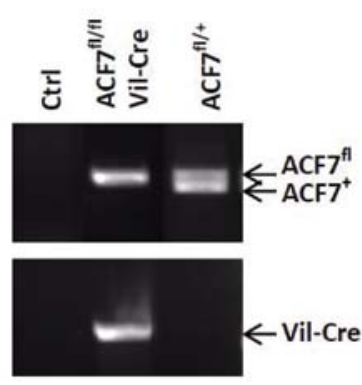

C

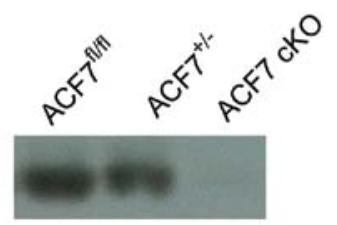

WB: ACF7
B

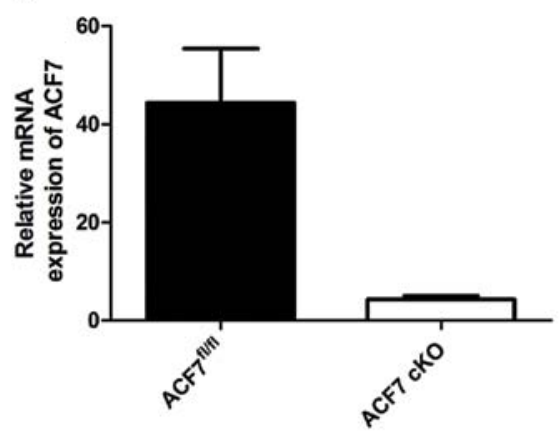

D $\quad$ ACF7

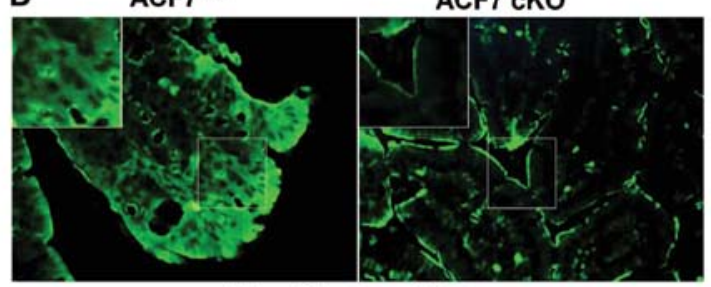

Magnification $400 \mathrm{X}$

Figure 1. ACF7 expression in the colonic mucosa of ACF7-cKO mice. (A) PCR genotyping of colonic mucosal samples from ACF7-cKO and ACF7 ${ }^{\mathrm{A} / \mathrm{f}}$ mice. (B) The relative mRNA level of ACF7 in colonic mucosa was decreased in ACF7-cKO mice when compared to the level in the control ACF7 ${ }^{1 / 1 / 1}$ mice (0.097-fold; $4.309 \pm 0.73$ vs. $44.36 \pm 11.08$ ) (Data are means \pm SEM; $P<0.01$, unpaired $t$-test; $n=5 /$ group). (C) Western blot analysis of ACF7 in colonic mucosal extracts revealed that ACF7 protein was decreased in the $\mathrm{ACF}^{+/}$mice and that no ACF7 protein was evident in the ACF7-cKO mice. (D) Fluorescence immunostaining showed weak ACF7 expression in the colonic mucosa of the ACF7-cKO mice compared to that in the ACF $7^{\mathrm{A} / \mathrm{fl}}$ mice.

The membranes were blocked for $1 \mathrm{~h}$ at room temperature in $5 \%$ non-fat dried milk, and then incubated with the primary antibody (1:500; Abcam, UK) with continuous gentle agitation overnight at $4^{\circ} \mathrm{C}$. The membranes were then incubated for $1 \mathrm{~h}$ with HRP-conjugated secondary antibodies (1:2,000; Beyotime, China) at room temperature, and finally developed by the ECL western blotting system (Thermo Scientific-Pierce, USA).

Immunofluorescence. Colonic tissue sections were incubated with anti-mouse occludin, anti-mouse claudin-1, anti-mouse ZO-1 (1:100; Abcam), and FITC-labeled goat anti-mouse IgG (1:50; Sigma, USA) was used as a secondary antibody. Typical images were captured using a DSY5000X microscope and Nikon D200 camera at a x400 magnification (excitation at $485 \mathrm{~nm})$.

Statistical analysis. Data are presented as means \pm SEM. The differences in mRNA levels of ACF7, ZO-1, occludin and claudin-1, between the ACF7-cKO and ACF $7^{\mathrm{fl} / \mathrm{fl}}$ were compared using an unpaired t-test. Differences in the FITCinulin filtration rates using a Ussing chamber were compared by monofactor ANOVA analysis (GraphPad, Prism, USA). $\mathrm{P}$-value below the level of $\alpha=0.05$ was considered to indicate a statistically significant result.

\section{Results}

Generation of conditional intestinal ACF7-cKO mice. By genotyping, we successfully identified ACF7-cKO mice (Fig. 1A). As expected, the relative level of ACF7 mRNA declined drastically in the colonic mucosa of the ACF7-cKO mice when compared to that in the control ACF $7^{\mathrm{fl} / \mathrm{fl}}$ mice (0.097-fold; $4.309 \pm 0.73$ vs. 44.36 $\pm 11.08 ; \mathrm{P}<0.01$, unpaired t-test; $n=5 /$ group) (Fig. 1B). Immunoblot analysis against the ACF7 protein showed that ACF7 protein was decreased in the colonic mucosa of the $\mathrm{ACF}^{+/-}$mice and no $\mathrm{ACF} 7$ protein was evident in the ACF7-cKO mice (Fig. 1C). Fluorescent immunostaining also showed weak ACF7 expression in the colonic mucosa in the ACF7-cKO mice compared to that in the control ACF $7^{\mathrm{f} / \mathrm{fl}}$ mice (Fig. 1D).

Rearrangement of mucosal epithelia and interstitial proliferation as detected by $H \& E$ staining. Through $\mathrm{H} \& \mathrm{E}$ staining, we demonstrated interstitial proliferation in the colonic sections of the ACF7-cKO mice. The results also revealed significant mucosal epithelial rearrangement in the ACF7-cKO mice when compared to the control (Fig 2).

Decreased colonic paracellular permeability in ACF7-cKO mice. We found a significant decrease in the colonic FITCinulin permeability rate between the $\mathrm{ACF} 7^{\mathrm{f} / \mathrm{fl}}$ and $\mathrm{ACF} 7-\mathrm{cKO}$ mice at $30 \mathrm{~min}(0.083 \pm 0.0085$ vs. $0.022 \pm 0.0086), 60 \mathrm{~min}$ $(0.104 \pm 0.0095$ vs. $0.052 \pm 0.0066), 90 \mathrm{~min}(0.116 \pm 0.0106$ vs. $0.057 \pm 0.0057)$ and $120 \mathrm{~min}(0.129 \pm 0.0106$ vs. $0.067 \pm 0.0083)$ (one-way ANOVA test; n=5/group) (Fig. 3).

Decreased occludin, claudin-1 and ZO-1 expression in ACF7-deficient colonic mucosa. Compared to controls, the relative quantitative value of mRNA expression of ZO-1, occludin and claudin-1 was significantly lower in the ACF7-cKO mice by 2.65 -fold $(16.79 \pm 2.146$ vs. $6.331 \pm 1.482$, Fig. 4A), 7.22-fold (348.4 \pm 95.35 vs. $48.27 \pm 5.889$, Fig. 4B) and 3.62 -fold ( $1.990 \pm 0.104$ vs. $0.550 \pm 0.157$, Fig. 4 C), respectively (unpaired t-test; $\mathrm{n}=5$ /group). Western blot analyses of the TJPs, $\mathrm{ZO}-1$, occludin and claudin-1, revealed a decrease in ZO-1, 


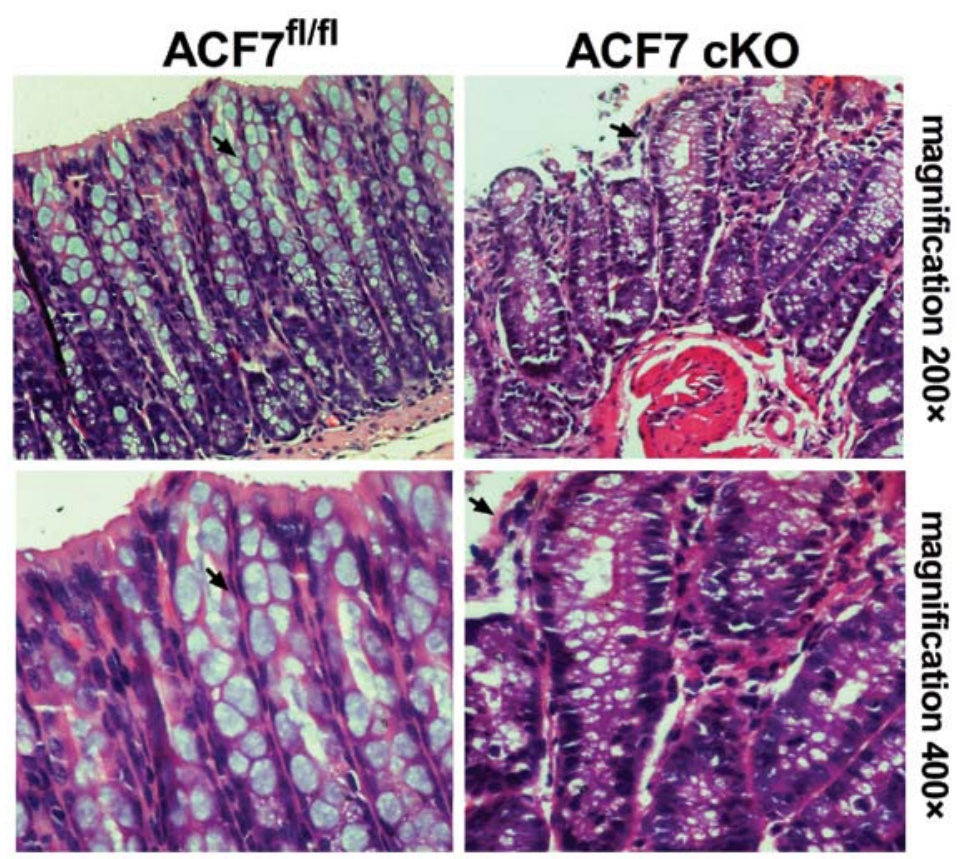

Figure 2. H\&E staining. Arrows show the same location of columnar epithelial colonic mucosal in the images of histopathological sections (x200 and $\mathrm{x} 400$ magnification). ACF7 cKO mouse samples show disrupted and squeezing mucosal epithelia and interstitial proliferation (x200 magnification). Columnar epithelial cell rearrangements are also shown (x400 magnification) in the colonic mucosa of the ACF7 cKO mice compared to that in the ACF7 $7^{\mathrm{f} / \mathrm{I}}$ mice.

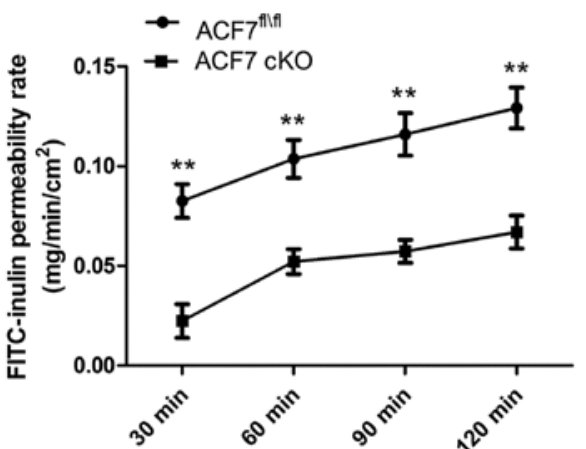

Time of colonic mucosa mounted in Ussing chambers

Figure 3. A significant decrease in the colonic FITC-inulin permeability rate was noted in the $\mathrm{ACF} 7-\mathrm{cKO}$ mice vs. the $\mathrm{ACF} 7^{\mathrm{fl} / \mathrm{fl}}$ mice at $30 \mathrm{~min}$ ( $0.083 \pm 0.0085$ vs. $0.022 \pm 0.0086), 60 \mathrm{~min}(0.104 \pm 0.0095$ vs. $0.052 \pm 0.0066)$, $90 \mathrm{~min}(0.116 \pm 0.0106$ vs. $0.057 \pm 0.0057)$ and $120 \mathrm{~min}(0.129 \pm 0.0106$ vs. $0.067 \pm 0.0083)$. All data were collected and expressed as means \pm SEM; one-way ANOVA test; $\mathrm{n}=5 /$ group; ${ }^{* *}<\mathrm{P}<0.01$.

occludin and claudin-1 expression in the colonic tissues of the ACF7-cKO mice vs. the ACF7 $7^{\mathrm{f} / \mathrm{fl}}$ mice (n=5/group) (Fig. 4D). Immunofluorescence staining further demonstrated that ZO-1, occludin and claudin-1 were significantly downregulated in the colonic mucosa of ACF7-cKO mice compared to levels in the $A C F 7^{\mathrm{f} / \mathrm{fl}}$ mice (Fig. 4E).

\section{Discussion}

ACF7 has recently been identified as a key integrator in cellular skeleton dynamics and cell mobility (16). Gene knockdown in an animal, and observation of the entire animal phenotype are the most effective methods of investigation of gene function (17-19). As gene knockout plays an important role during development, traditional knockout sometimes leads to the death of the embryo. Therefore, cKO utilizing the Cre-loxP strategy may avoid this problem (20). Since the ACF $7^{-/-}$mouse displays fetal lethality (11), we chose to establish a colonic mucosa ACF7-cKO mouse model. Results of our Ussing chamber study indicated a significant decrease in the colonic mucosal permeability in ACF7-cKO mice. H\&E staining of the colonic mucoca indicated a positive association between the microscopy findings and cytoskeleton dysregulation. By H\&E staining, we observed that epithelial arrangement was disturbed in the ACF7-deficient colonic mucosa, and we hypothesized that cytoskeleton dysregulation results in an alteration of colonic paracellular permeability. Ussing chamber and FITC-inulin results of the colonic paracellular permeability were also correlated with qRT-PCR, western blotting and immunofluorescence results for ZO-1, occludin and claudin-1. The significant decrease in these proteins indicated that TJPs were correlated with ACF7 expression.

The interaction of epithelial TJPs with cytoskeleton proteins has been recognized as essential. The tight junctions can rapidly adapt, in response, to diverse external signals via structural and functional linkage between TJPs and cytoskeleton proteins (21). Tight junctions are the most apical organelles of the apical junctional complex and are primarily involved in the regulation of paracellular permeability. Although previous researches have concerned with the individual molecules of the tight junctions as well as their mutual interactions. Only recently has it been noted to what extent TJPs are important for the regulation of intestinal permeability and which proteins are involved.

Occludin, claudin-1 are transmembrane proteins. They are critically involved in the regulation of the TJ barrier (22). Previous studies have shown that the expression of occludin and claudin-1 is tightly associated with intestinal paracellular permeability (23). Occludin expression levels correlate with 

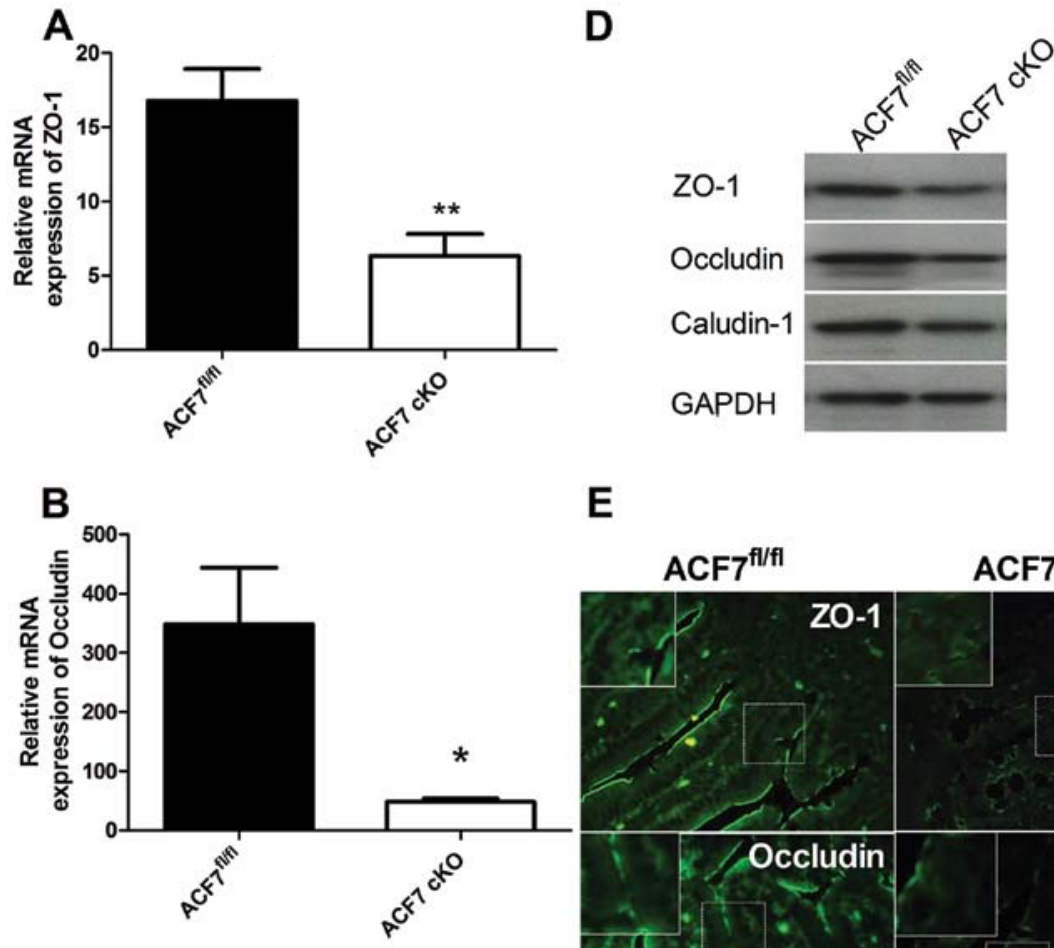

E
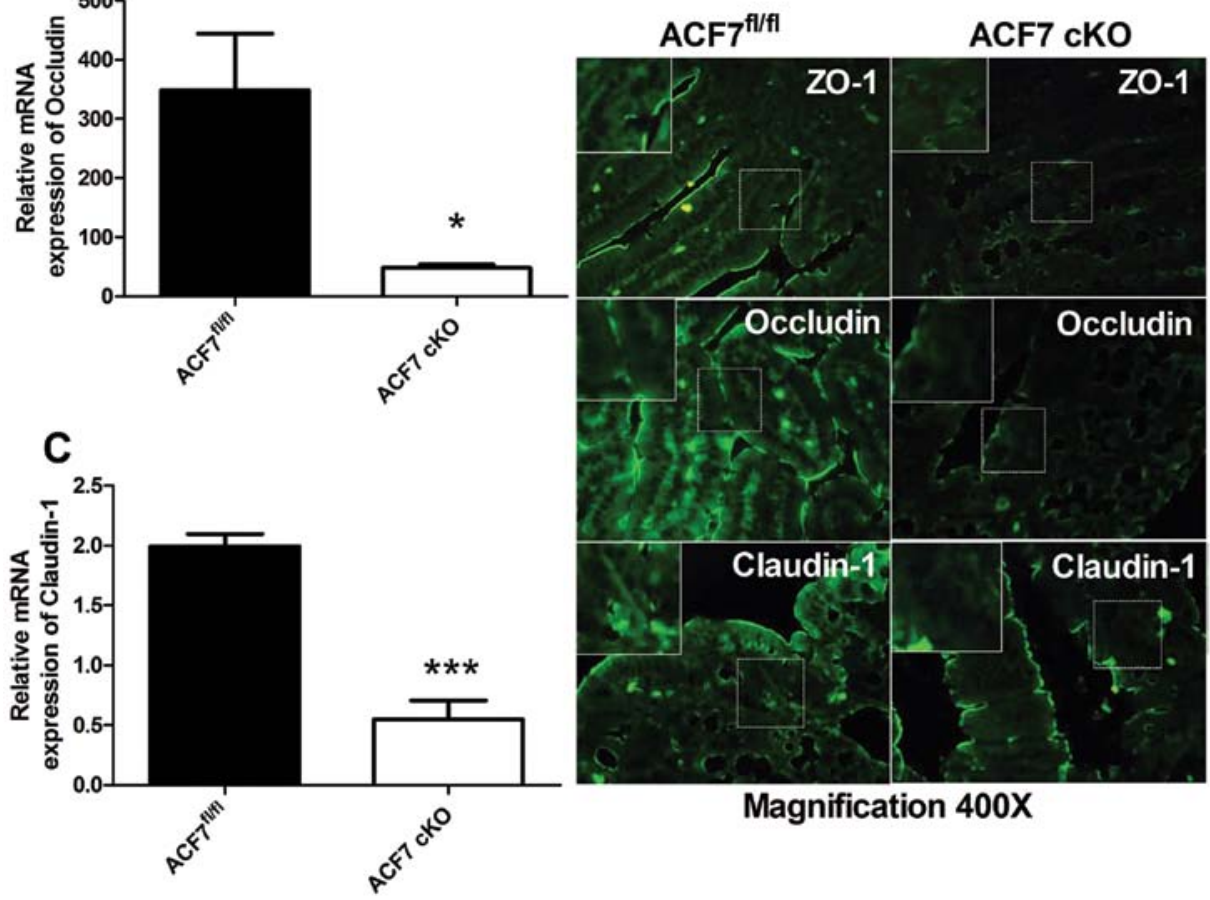

Figure 4. qRT-PCR results indicate that expression of ZO-1, occludin and claudin-1 is significantly lower in the colonic mucosa of ACF7-cKO mice

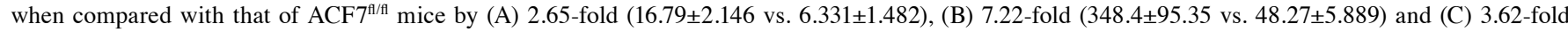
( $1.990 \pm 0.104$ vs. $0.550 \pm 0.157)$, respectively. All data are collected and expressed as means $\pm \mathrm{SEM}$; unpaired $\mathrm{t}$-test, $\mathrm{n}=5 /$ group; ${ }^{*} 0.01<\mathrm{P}<0.05,{ }^{* *} 0.001<\mathrm{P}<0.01$, ${ }^{* * *} \mathrm{P}<0.001$. (D) A decrease in ZO-1, occludin and claudin-1 expression in colonic tissues of ACF7-cKO mice vs. ACF $7^{\mathrm{n} / \mathrm{fl}}$ mice was noted ( $\mathrm{n}=5 / \mathrm{group}$ ). (E) Immunofluorescence staining at a x400 magnification is shown. In ACF7/1/1 and ACF7-cKO mice, ZO-1, occludin and claudin-1 are expressed in green fluorescence. ACF7-cKO mouse samples show significant downregulation of all three TJPs in the colonic epithelium.

the number of tight junction strands in varied colonic epithelia permeability. Occludin was found to be associated with permeability for ions and small solutes in siRNA experiments (24). In contrast, the role of occludin in intestinal paracellular permeability has not been recognized until one report revealed that occludin-deficient mice exhibit normal epithelial and paracellular permeability (25). The family of claudin proteins consists of a number of essential components associated with deleterious barrier impairment in the event of their deficiency (26). For example, claudin-1-deficient mice died within $24 \mathrm{~h}$ of birth, and further study found dramatic fluid and electrolyte loss through intestinal mucoca and skin (27). It has been shown in inflammatory bowel disease (IBD) that decreased ZO-1 expression is associated with intestinal paracellular permeability (28). ZO-1 is a peripheral membrane protein that binds to both the $\mathrm{C}$ terminal of occludin and F-actin, thus stabilizing the cytoskeleton structure (29). ZO-1 connects to strand-forming TJPs with cytoskeletal proteins (actin and microfilaments) (30). One animal model with upregulation of TJPs showed a decrease in intestinal paracellular permeability under GvHD condition (31). Thus, there are different points of view and some are paradoxical.

The previously mentioned studies reflect one important fact. Since these models are established on a complex physiological shock condition and lack long-term homeostasis, the results are varied. Thus, we chose to establish ACF7-knockout mice instead, generating a homogeneous and stable model with which to provide strong evidence for a change in paracellular permeability. Consequently, based on these findings, ACF7 alters the mucosal epithelial arrangement, colonic paracellular permeability and regulates tight junction protein expression. The ACF7-cKO mouse is a suitable model with which to study intestinal permeability regulatory mechanisms and related diseases. 


\section{Acknowledgements}

The authors acknowledge Dr Xiaoyang Wu of the Howard Hughes Medical Institute and Laboratory of Mammalian Cell Biology and Development, Rockefeller University, New York, NY, USA for his kind donation of ACF7 $7^{1 / 1 / 1}$ mice; Professor Dazheng Wu of the Shanghai University of TCM for supplying the facilicities for Ussing Chamber test and his assistance in the research; Sibo Zhu of the CinoAsia Institute, Shanghai, China for his techinical guidance. This study was supported by three grants from Medical School of Shanghai Jiaotong University PhD Inovation Funding (BXJ201236), LIJIESHOU Funding (LJS_201108) and NFSC Funding (81070293).

\section{References}

1. Catalioto RM, Maggi CA and Giuliani S: Intestinal epithelial barrier dysfunction in disease and possible therapeutical interventions. Curr Med Chem 18: 398-426, 2011.

2. Hossain $\mathrm{Z}$ and Hirata T: Molecular mechanism of intestinal permeability: interaction at tight junctions. Mol Biosyst 4 1181-1185, 2008 .

3. Gasbarrini G and Montalto M: Structure and function of tight junctions. Role in intestinal barrier. Ital J Gastroenterol Hepatol 31: 481-488, 1999.

4. Ulluwishewa D, Anderson RC, McNabb WC, Moughan PJ, Wells JM and Roy NC: Regulation of tight junction permeability by intestinal bacteria and dietary components. J Nutr 141 769-776, 2011

5. Shen L, Su L and Turner JR: Mechanisms and functional implications of intestinal barrier defects. Dig Dis 27: 443-449, 2009

6. Madara JL, Stafford J, Barenberg D and Carlson S: Functional coupling of tight junctions and microfilaments in T84 monolayers. Am J Physiol 254: G416-G423, 1988.

7. Watanabe T, Noritake $\mathrm{J}$ and Kaibuchi K: Regulation of microtubules in cell migration. Trends Cell Biol 15: 76-83, 2005.

8. Tsvetkov AS, Samsonov A, Akhmanova A, Galjart N and Popov SV: Microtubule-binding proteins CLASP1 and CLASP2 interact with actin filaments. Cell Motil Cytoskeleton 64: 519-530, 2007.

9. Kodama A, Karakesisoglou I, Wong E, Vaezi A and Fuchs E: ACF7: an essential integrator of microtubule dynamics. Cell 115: 343-354, 2003.

10. Ghandehari H, Smith PL, Ellens H, Yeh PY and Kopecek J: Size-dependent permeability of hydrophilic probes across rabbit colonic epithelium. J Pharmacol Exp Ther 280: 747-753, 1997.

11. Chen HJ, Lin CM, Lin CS, Perez-Olle R, Leung CL and Liem RK: The role of microtubule actin cross-linking factor 1 (MACF1) in the Wnt signaling pathway. Genes Dev 20: 1933-1945, 2006.

12. Wu X, Kodama A and Fuchs E: ACF7 regulates cytoskeletalfocal adhesion dynamics and migration and has ATPase activity. Cell 135: 137-148, 2008.
13. Arrieta MC, Madsen K, Doyle J and Meddings J: Reducing small intestinal permeability attenuates colitis in the IL10 genedeficient mouse. Gut 58: 41-48, 2009.

14. Baker NT and Graham LL: Campylobacter fetus translocation across Caco-2 cell monolayers. Microb Pathog 49: 260-272, 2010.

15. Volpe DA: Application of method suitability for drug permeability classification. AAPS J 12: 670-678, 2010.

16. Wu X, Shen QT, Oristian DS, et al: Skin stem cells orchestrate directional migration by regulating microtubule-ACF7 connections through GSK3 $\beta$. Cell 144: 341-352, 2011.

17. Austin CP, Battey JF, Bradley A, et al: The knockout mouse project. Nat Genet 36: 921-924, 2004.

18. Brown SD and Hancock JM: The mouse genome. Genome Dyn 2: 33-45, 2006

19. Dinnyes A and Szmolenszky A: Animal cloning by nuclear transfer: state-of-the-art and future perspectives. Acta Biochim Pol 52: 585-588, 2005.

20. Kühn R and Schwenk F: Advances in gene targeting methods. Curr Opin Immunol 9: 183-188, 1997.

21. Madara JL, Barenberg D and Carlson S: Effects of cytochalasin D on occluding junctions of intestinal absorptive cells: further evidence that the cytoskeleton may influence paracellular permeability and junctional charge selectivity. J Cell Biol 102: 2125-2136, 1986.

22. Tsukita S and Furuse M: Occludin and claudins in tight-junction strands: leading or supporting players? Trends Cell Biol 9: 268-273, 1999.

23. Ye D, Guo S, Al-Sadi R and Ma TY: MicroRNA regulation of intestinal epithelial tight junction permeability. Gastroenterology 141: 1323-1333, 2011.

24. Yu AS, McCarthy KM, Francis SA, et al: Knockdown of occludin expression leads to diverse phenotypic alterations in epithelial cells. Am J Physiol Cell Physiol 288: C1231-C1241, 2005.

25. Schulzke JD, Gitter AH, Mankertz J, et al: Epithelial transport and barrier function in occludin-deficient mice. Biochim Biophys Acta 1669: 34-42, 2005.

26. Furuse $M$ and Moriwaki K: The role of claudin-based tight junctions in morphogenesis. Ann NY Acad Sci 1165: 58-61, 2009.

27. Furuse M, Hata M, Furuse K, et al: Claudin-based tight junctions are crucial for the mammalian epidermal barrier: a lesson from claudin-1-deficient mice. J Cell Biol 156: 1099-1111, 2002

28. Piche T, Barbara G, Aubert P, et al: Impaired intestinal barrier integrity in the colon of patients with irritable bowel syndrome: involvement of soluble mediators. Gut 58: 196-201, 2009.

29. Keon BH, Schäfer S, Kuhn C, Grund C and Franke WW: Symplekin, a novel type of tight junction plaque protein. J Cell Biol 134: 1003-1018, 1996.

30. Fanning AS and Anderson JM: Zonula occludens-1 and -2 are cytosolic scaffolds that regulate the assembly of cellular junctions. Ann NY Acad Sci 1165: 113-120, 2009.

31. Noth R, Lange-Grumfeld J, Stüber E, et al: Increased intestinal permeability and tight junction disruption by altered expression and localization of occludin in a murine graft versus host disease model. BMC Gastroenterol 11: 109, 2011. 